\title{
A study with Paperless Electronic Medical Advice
}

\author{
Sheng Li HU ${ }^{1}$, a , Jin Qing $\mathrm{CHI}^{2}$ \\ ${ }^{1}$ Central Hospital of Cangzhou, Information Center, Hebei Province, China \\ ${ }^{2}$ Central Hospital of Cangzhou, Bidding office, Hebei Province, China
}

\begin{abstract}
The purpose of this study is to drive the EMR's further application and rapid expansion clinically based on the electronic medical advice as the core thereby realizing the paperless EMR. This paper has analyzed the implementation effects of electronic medical advice, followed by discussion of frequently asked questions (FAQ) and development direction, and finally analyzed the solution of paperless EMR. It indicates that electronic signatures and unitrust time stamp are keys to achieve the paperless EMR. The laws and regulations, new ideas, paperless consultation sheets, all data integration and sharing, virtual printing technology, application of wireless and mobile ward-round trolley, etc. within the health care field shall be proposed to be improved. It is significantly important to save the medical costs, reduce the medical negligence, achieve the paperless EMR and build a high-quality digital hospital.
\end{abstract}

\section{The advantages of Paperless Electronic Medical Advice}

\subsection{Background}

It is an important content to deepen the public hospital reform by establishing and completing the hospital information system (HIS) based on the Electronic Medical Record (EMR) as the core. It has important meanings in promotion of medical service equalization; driving the medical service management to be developed towards scientification, specialization and informatization; enhancing the hospital's work efficiency and protecting the medical safety. Electronic medical advice is a core of EMR, so the construction and development of electronic medical advice system are extremely important.

\subsection{Effect analysis of electronic medical advice implementation}

Electronic medical advice is an important part of EMR and a core business of hospitals. It is not only a proof of treatment settlement, but also legal evidence. From the implementation effects we can see, the electronic order system can optimize the clinical working process thereby reducing the workload of medical staff, improving work efficiency, and guaranteeing the medical safety [1-12].

For doctors, electronic medical advice can make the medical records clear, neat, standard thereby avoiding the medical hidden dangers due to poor handwriting [1]. According to different symptoms, the medical advice is maintained differently. The medical advice is automatically linked to the corresponding charges. The

\footnotetext{
a Corresponding author: hushenglihsl@139.com
}

contents of those charges consist of standard charging codes and charging names that are ticked in the saved medical advice database when using. It can directly enter into the medical advice interface. This can save plenty of time in making the medical advice; therefore, doctors will have more time to communicate with patients.

For nurses, the medical risks made by copying the medical advice to the treatment book, injection book, oral administration book, inspection book could be reduced. Automatic charging begins after medic advice received. Those fees ac-cord with the commodity price and no need to add the costs again, therefore, nurses can get more time to deliver the humanized warm care to patients and patient's satisfaction will be improved.

For mangers, the medical advice can be improved. In this system, the trade name or common name can be used for searching. However, finally the drug common name, patent medicine name of new active compounds and compound preparation name that meet the prescription regulations will be submitted. This will avoid a wrong trade name occurred in the medical advice. When submitting the medical advice, the usage, dosage and departments should be checked throughout in order to guarantee the accuracy of medical advice, as well as avoid the potential risks resulting from signature missing.

\section{Frequently Asked Questions and Countermeasures in Electronic Medical Advice}

\subsection{Frequently asked questions}


In the implementation of electronic medical advice, it may have all kinds of mistakes either man made or technical defects. More common problems include injection dosage, specification, unit mistakes, confused long-term and temporary medical advice, inconsistent performers and signatures, and discrepancy between stopping time and actual time resulting in overcharge and charging missing, etc.

\subsection{Countermeasure analysis}

First from the side of management, on the one hand, medical staff should enhance the training in laws and regulations and reinforce the legal awareness; on the other hand, the management system of electronic medical advice should be completed and effective rewards and punishments should be set out. A communication coordination mechanism should be established among doctors, nurses, pharmacists, laboratories' and inspectors in order to ensure a smooth communication and avoid unnecessary medical disputes. When performing rescuing tasks, relevant medical advice and expenses should be additional recording without delay in order to ensure the accuracy of medical advice and expenses. Nurses have responsibilities on supervision of doctor's diagnosis and treatment scheme and the medical advice should be implemented after seriously checked. Meanwhile, the advanced methods of other hospital's medical advice should be studied in order to use the echoing check method making nurses mutual supervision, improving the mistake detection rate, and preventing occurrence of errors. To establish a special treatment book and nurse hand-over book; faithfully implement checking system; be standard in charging; and avoid overcharge and missing charge[2]. After nurses received and processed the medical advice, if they have to leave for something, the job number must be withdrawn in time in case unnecessary troubles.

Secondary from the view of technical means, to complete the electronic medical advice system and avoid the some mistakes from technical level, such as missing signatures, non-received and unprocessed orders, and the discharge notification and ward transfer won't be dealt with. Only if those medical orders are processed, the next operation will take place.

\section{Development trend of electronic medical advice}

\subsection{Paperless EMR is based on the electronic medical advice as the core}

By studying the published documents and development trend of medical informationization in recent years, we have found that electronic medical advice system is combined with data mining and artificial intelligence technology developing towards paperless, intelligent and standard.

It is in a starting stage. Paperless management will be achieved in long-term and temporary medical advice, progress notes, various kinds of special format records, all kinds of tests and examination application forms.

\subsection{Intelligent electronic medical advice}

When making new medical advice, stopping medical advice and cancelling medical advice, there is an automatic dialog box popped up at nurse station, intelligently reminding nurses to receive and perform. When medical advice is stopping, it is also stopping charging, which will avoid extra charge caused by nurses forget stopping the medical advice.

\subsection{Electronic medical advice standardization}

Standardization for medical advice, especially, the standardization management is used for medical nonmedication and examination advice. For example, discharged next day and discharge tomorrow, etc. shall be normalized as discharge tomorrow. This is convenient for quality control of electronic medical advice. If there is in no medical advice of discharge tomorrow, the discharge notice cannot be processed. Through medical advice standardization, the medical advice quality can be improved via technological means.

\section{Paperless emr with electronic medical advice as the core}

\subsection{Issues paperless EMR faced}

At present, most of hospitals are still in a completely paper medical record stage or paper and paperless medical record coexisting administrative stage. Due to paperless EMR has many advantages of compared with paper medical record and it is also an important symbol of digital hospital and is an inevitable trend of modern hospital management. We will discuss about issues that paperless EMR is faced, as well as solutions.

From article we can see, paperless EMR is facing many problems, including imperfectly related laws and regulations, incapably guaranteed safety of electronic signatures, without establishing certificate authority (CA) within health care field, without choosing qualified unitrust Time Stamp Authority (TSA) etc. The hysteretic laws and regulations restrict the development of paperless EMR. Moreover, the ideas of hospital managers falling behind and the costs of medical informationization difficult to shoulder are also factors to barrier the paperless EMR.

\subsection{Electronic signatures and unitrust time stamp}

When patients print the long-term and temporary medical advice after discharge, for purpose of medical advice is accordance with norm of law, printed paper medical advice sheet must be signed in handwriting. Medical advices are involving in numerous medical staff; review signatures waste time and energy and easily go wrong. 
Therefore, using electronic signatures and unitrust time stamp replacing hand-written signatures is a key to achieve the paperless EMR. Apart from using the electronic signatures in the paperless EMR, medical staff must retain all previous amendment trace and time in the EMR when amending the medical records. It is predicted that in the near future, along with improvement of electronic signatures, unitrust time stamp and related laws and regulations, paperless EMR will enter into an integrated development period.

\subsection{Update ideas}

To further improve various rules and regulations within the health care field, make the EMR be in accordance with laws and regulations, and solve the lagging legal system resulting from application of new technology. In addition, hospital managers shall also update the new ideas, advance with times, and properly increase the input in medical informationization.

\subsection{Consultation issues}

Doctors will sign their names after they make a medical advice order, the consultation form in the order will be delivered to a relevant department, medical staff in those related departments will confirm after seeing. The relevant consultation notes will be filled after consultation termination. Experts who take part in consultation will process the electronic signatures [3]. The entire process can realize the paperless management.

\subsection{Integration and sharing of all system data}

Conclusions EMR should achieve the data interface with HIS, PACS, LIS, operation anesthesia, physical examinations, electronic application form, etc. The information sharing is achieved based on the data integration. All information related to patients in the EMR, including various test and examination results, ultrasound, CT imaging, operation anesthesia records and videos, etc. At this time, EMR is a multimedia EMR, including text, sound and images, etc. With respect to system integration scheme, HL7 interface mode is recommended to use for effective integration. The Drug Consultation and Drug Safety Detection System shall be implanted into the EMR. The overdose, contraindication and incompatibility will be remained intelligently to guide the rational medications for doctors when recording the medical advice in order to prevent the phenomenon of irrational drug use [4].

\subsection{Virtual print technology}

Take advantages of virtual printing technology can directly converse all kinds of data information in the EMR into to PDF, JPG, TIF, BMP, PCX, PNG format. Then those format data files will be saved in the hard disks or light disks by binary forms after they are encrypted. Other application programs cannot open those medical records, which guarantees medical data unable to be amended thereby realizing EMR data off-line storage and working out the problems of system performance decline caused by infinitely data increasing in the database as well as an important step of achievement of paperless EMR filing.

\subsection{Applications of wireless mobile ward-around trolley}

Doctors can carried out the ward-around by wireless computer ward-around trolley in each ward via deploying the WLAN and using the barcode technology. They can directly make medical advice, check various kinds of images such as CT, CR image device and all test results. Nurses can directly view medical advice information and execution as well as type-in patient's indicators. The application of wireless mobile ward-around trolley is an important milestone of realization of paperless EMR.

\subsection{All self-service devices shall be placed in a notable place of hospitals}

After implementing EMR, for the sake of maximum protection of patient's right to know, hospitals shall place various kinds of self-service devices at a notable place. This will let patients check the expense details and conduct a satisfaction evaluation for the entire treatment. This is very important in easing the current tension of doctor-patient relationship and creating a harmonious hospital.

\subsection{Paper documents}

At the present, EMR is unable to be really paperless, namely there are still some paper medical records, such as consent for operation that is needed relatives of patients signing the documents, some independent devices such as cerebral electrical device that provides the paper reports due to unable to access the network because of interface problems.

\subsection{Set out an emergency response for paperless medical record}

For some problems occurred during the paperless medical record, an emergency response shall be set out, such as network paralysis caused the system unable to be normal operation, frequent electronic signatures lead to the KEY often changing, the temporary KEY unable to be made up by the third party certificate authority due to weekends or nights, all of which may cause medical staff unable to login resulting in patients waiting for long time.

\section{Conclusion}

Paperless EMR is still in starting stage; many aspects are still imperfect and need us to constantly make great efforts thereby striving for early implementation of real paperless EMR and construction of E-hospitals. 
The research work was supported by Cangzhou Science and Technology Research and Development Program guidance. In the process of paper writing, my friend Zhi Guo gave the vigorous help and put forward much valuable and impartial advice, and at the same time, expresses my heartfelt thanks to the experts who revised my paper.

\section{References}

1. Cao Yang, Wang Yan. Optimization of Doctor's Order Inputs inter-face and functions of Anamnesis. Chinese Journal of Medical instrumentation, 34, 5, (2010).

2. Cao Yang, Ding Aimin, Wang Yan. New Model of Doctor-Nurse Communication Based on Electronic Medical Advice Platform. Chinese Journal of Medical instrumentation, 36, 1, (2012).

3. Chen Xiaohua, Wang Li, Chen Qun. Electronic check prescription application submission work. Journal of Nursing Science, 26, 7, (2011).

4. Gu hui. Defect analysis and countermeasures in the process of executing electronic doctor's advice by nurses. Journal of Clinical Medicine in practice, 15, 22, (2011).

5. Hu Shengli, Bai Hongmin. Research on electronic signatures application in electronic medical records. Chinese Hospital, 15, 11, (2011).

6. Li Wei, Li Fengsheng, Wang Qisheng.Analysis based on digital trans-mission system of hospital construction under doctor's orders, Hospital administration Journal of Chinese People's liberation Army, 17,10, (2010).

7. Tao Hongyan. ICU nurses prevent the implementation of response measures electronic prescription errors. National Medical Frontiers of China, 16, (2011).

8. Xie Haiying, Li Ni, Yan Chengmei etc. Effectiveness of Printing and Bedside Use of Medical Orders in Nursing Work. Nursing Journal of Chinese People's Liberation Army, 25,4B, (2008).

9. Yang Li, Sun Sunchun.Cooperation of doctor and nurse solving faculty electronic medical order input question. China Medicine Guides, 18, 25, (2014).

10. Yue LI, Zhang Xiaoling, Guo Lipingl. New doctor checking method in the electronic Prescription. Yiyao Qianyan, 12, 13, (2012).

11. Zhang Danjing. PDA application experience in clinical nursing. Chinese Nurse Management9, 3, (2009).

12. Zheng Xichuan, Tan Shensheng, Wu Yunzhen. Implementation the computerized Provider order Entry through Optimizing Clinical Workflow. Chinese Digital Medicine, 7, 3, (2011). 